\title{
Effects of Apple and Pear Wood Vinegar Components on Pleurotus ostreatus Mycelium Growth
}

\begin{abstract}
Hao Xu, ${ }^{\mathrm{a}, \mathrm{b}}$ Jian Zhao, ${ }^{\mathrm{a}}$ Yang Jing, ${ }^{\mathrm{a}}$ Jingcong Xie, ${ }^{\mathrm{a}}$ Ning Zhang, ${ }^{\mathrm{a}}$ and Jianchun Jiang ${ }^{\mathrm{a}, *}$
In order to facilitate the application of wood vinegar in the mushroom industry, a framework was developed to reveal the individual and interactive effects of chemical groups in wood vinegars on Pleurotus ostreatus mycelium growth. By a series of refining and separating methods, the crude wood vinegar samples were processed and separated into six subgroups with distinctive component concentrations in each. Adding the wood vinegar subgroups into the culturing medium resulted in differences in mycelium growth. Analysis of variance was performed on the differences to evaluate the effects of seven chemical groups on mycelium growth. The enhancing effects of groups of chemicals were (ranked by effect) alcohols > esters > aldehydes; the inhibiting groups of chemicals were phenols $>$ ketones $>$ acids. The principle inhibitory chemicals in the wood vinegars were most likely 1,2-benzenediol, 2methyl phenol, and 4-ethyl-2-methoxyphenol. The synergistic effects between acids and phenols and between acids and ketones were confirmed. By these effects, the inhibiting chemicals interacted synergistically as mycelium growth promoters.
\end{abstract}

Keywords: Wood vinegar; Pleurotus ostreatus; Synergistic effect

Contact information: a: Institute of Chemical Industry of Forest Products, CAF, Nanjing 210042, China; b: National Engineering Laboratory for Biomass Chemical Utilization, Nanjing 210042, China;

* Corresponding author: Jiangjianchun@icifp.cn

\section{INTRODUCTION}

For years, the mushroom industry has been seeking a cheap and environmentally friendly culturing agent that not only can prevent/control diseases and pests but also promote mushroom growth. Wood vinegar is a water-soluble by-product collected from the flue during wood carbonization. As a broad-spectrum, easy-to-obtain synergist of edible fungi, it has been widely considered as potential culturing synergist in the industry since the middle of the last century (Burnette 2010). However, due to the complexity of wood vinegar, there has been no industrial-scale application of the compounds reported. According to analysis, wood vinegar generally contains about 300 kinds of compounds, such as acetic acid (3\% to 8\%), formic acid (approximately 0.5\%), methanol (approximately 1\%), formaldehyde (approximately 1\%), acetone (approximately 1\%), and guaiacol $(0.1 \%$ to $0.5 \%)$. Because the chemical components are highly related to the kinds of raw materials, the growth stages, and the carbonization conditions, among many other factors, the components of wood vinegars are highly complex. This complexity has resulted in ambiguous or even contradictory conclusions among some previous studies on the effects of wood vinegars on edible fungi.

Among wood vinegar components, 3,5-dimethylphenol, 2-methoxyphenol, butanoic acid, and 1-pentanol have significant effects in promoting the mycelium growth and/or fruiting body formation of edible fungi (Ikegaya et al. 1994; Yoshimura et al. 1995; Singh et al. 2018). However, a wood vinegar sample generally contains about 300 kinds of 
compounds, for which the concentration of each analyzable compound was within the range from $0.05 \%$ to $1 \%$. Therefore, it has proved difficult to isolate individual compounds from such mixtures. Practically, separating the crude wood vinegar by basic refining and separating methods into mixture compounds could be promising. Furthermore, there have also been speculations in the industrial community that the growth-enhancing effects of wood vinegars are not limited to individual compounds but are also derived from synergistic effects from a variety of active substances. Nevertheless, due to the complex nature of the problem, the information needed to draw a conclusion on these synergistic effects among different components has been far from sufficient. Up to now, few studies have looked at this issue. Thus, the industrial application demands a feasible and reliable method to determine whether the synergistic effects were present or not, and more importantly, if the said effects are present, by which combination of the components could the maximum promotion of mycelium growth be achieved. Besides, it is known that chemicals of certain group, such as the phenols, are potentially inhibitory for microorganism growth. Considering that phenol components account for $10 \%$ to $20 \%$ (Wu et al. 2015) of the unprocessed wood vinegars by weight concentration, depending on the raw materials and processing condition, it is only prudent to draw a conclusion on whether this group of chemicals should/could be removed before any industrial application.

To address these issues, in this study a series of refining and separating processes were combined with statistical inference to reveal the presence and characteristics of enhancing and inhibiting chemicals in original wood vinegars. In addition, analysis of variance (ANOVA) validated that synergistic effects exist between acids and phenols as well as between acids and ketones, and by these effects the former inhibiting chemicals interacted synergistically as mycelium growth promoters.

\section{EXPERIMENTAL}

\section{Microorganism and Inoculum Preparation}

The Pleurotus ostreatus strain used in this study was CICC 14012, supplied by the China Center of Industrial Culture Collection (CICC) (Beijing, China). The strain was grown and maintained on Czapek-Dox medium at $25^{\circ} \mathrm{C}$ for $7 \mathrm{~d}$. Shortly before inoculation, the mycelium was washed off from the Petri dish with $10 \mathrm{~mL}(5 \mathrm{~mL}+3 \mathrm{~mL}+2 \mathrm{~mL})$ of deionized water. The suspension was centrifuged at $8000 \mathrm{rpm}$ for $3 \mathrm{~min}$ to break down the mycelium, and then it was blended and sifted through a 40-mesh sieve. The filtered suspension was shaken briefly for homogenization and then pipetted into $1.5-\mathrm{mL}$ Eppendorf tubes with $0.2 \mathrm{~mL}$ in each for inoculation.

\section{Preparation and Refinement of Pyrolyzed Wood Vinegar}

Apple (Malus domestica) and pear (Pyrus spp.) wood was collected from orchards in Yishui, Shandong province, China. The collected wood was ground into sawdust and sieved through a 20 mesh screen. The sawdust was desiccated at $60{ }^{\circ} \mathrm{C}$ for $24 \mathrm{~h}$ and then analyzed for cellulose, hemicellulose, and xylose contents by standard measurement (Ghose 1987) published by National Renewable Energy Laboratory (NREL). The cellulose, hemicellulose, and xylose contents in apple wood were $44.2 \%, 23.8 \%$, and $24.9 \%$, respectively; in pear wood they were $41.9 \%, 19.2 \%$, and $26.5 \%$ respectively.

Twenty g of sawdust was placed into a quartz tube furnace $(500 \mathrm{~mL}$ volume $)$ under the protection of nitrogen flow $(250 \mathrm{~mL} / \mathrm{min})$. The sawdust was pyrolyzed with a 
programmed temperature increase of $20^{\circ} \mathrm{C} / \mathrm{min}$ and held at $450{ }^{\circ} \mathrm{C}$ for $30 \mathrm{~min}$. During the process, the volatile components were condensed and collected. The same process was repeated 3 times over, and the collected condensates (crude wood vinegar) from 3 repeats were blended for refinement.

For crude apple wood vinegar, after a $20-\mathrm{d}$ standing and layering process, the supernatant was siphoned and marked as A1; A1 was treated with activated carbon ( $2 \mathrm{wt} \%)$ for absorption treatment ( $2 \mathrm{~h}$ mixing and $24 \mathrm{~h}$ standing) and marked as A2. Then, A1 was decompression distilled, and the lower-boiling-point (less than $97{ }^{\circ} \mathrm{C}$ ) components were marked as A3, which accounted for $10 \%$ of the volume of A1. The intermediate-boilingpoint $\left(97{ }^{\circ} \mathrm{C}\right.$ to $\left.100{ }^{\circ} \mathrm{C}\right)$ components were marked as $\mathrm{A} 4$, which accounted for $70 \%$ of the volume of $\mathrm{A} 1$. The greater-boiling-point $\left(100^{\circ} \mathrm{C}\right.$ to $\left.105^{\circ} \mathrm{C}\right)$ components, which accounted for $20 \%$ of the volume of $\mathrm{A} 1$, were marked as A5. Five $\mathrm{mL}$ of A1 was extracted with 10 $\mathrm{mL}(5 \mathrm{~mL}+3 \mathrm{~mL}+2 \mathrm{~mL})$ of ethyl acetate, and then $5 \mathrm{~mL}$ of deionized water was added into the extracts, and the mixture was decompression distilled at $80^{\circ} \mathrm{C}$ for $15 \mathrm{~min}$ to remove ethyl acetate, with the remaining components marked as A6.

The same process was applied with crude pear wood vinegar, and the corresponding components were named accordingly (P1, P2, P3, P4, P5, and P6).

\section{Determination of Chemical Components}

The water contents of the samples were determined by Karl Fischer's method (Yatagai et al. 2002). The components of samples were analyzed by gas chromatographymass spectrometry (GC-MS) (7890A/5975C, Agilent, Santa Clara, CA, USA). The column was glass capillary with a film of cross-linked polyethylene glycol $(30 \mathrm{~m} \times 0.25 \mathrm{~mm} \times 0.25$ $\mu \mathrm{m})$. The gas chromatography process started at $50{ }^{\circ} \mathrm{C}$, which was held for $2 \mathrm{~min}$. Then, the temperature was increased to $280{ }^{\circ} \mathrm{C}$ at a rate of $5{ }^{\circ} \mathrm{C} / \mathrm{min}$ and held for $20 \mathrm{~min}$. The split ratio injection was 100:1, the sample size was $0.2 \mu \mathrm{L}$, and the carrier gas was helium with a flow rate of $1.0 \mathrm{~mL} / \mathrm{min}$. The mass spectrometry mode was electron ionization (70 $\left.\mathrm{eV}, 230{ }^{\circ} \mathrm{C}\right)$.

\section{Cultivation and Measurement of Biomass}

Ten mL of Czapek-Dox medium solution (sieved through 140 mesh) was placed in a $100-\mathrm{mL}$ shake flask as a basal culture medium. Then, $5 \mu \mathrm{L}$ to $17 \mu \mathrm{L}$ (fixed by the water content of each sample, as shown in Table 1) of the prepared wood vinegar samples was added into the flasks according to the design of the experiment. Then, $0.2 \mathrm{~mL}$ of prepared mycelium inoculum was added to each flask and cultivated at $25^{\circ} \mathrm{C}$ in the dark for $9 \mathrm{~d}$. After cultivation, the medium was sieved through 140 mesh. The collected mycelium was centrifuged twice at $8000 \mathrm{rpm}$ for $5 \mathrm{~min}$ each and then dried at $50{ }^{\circ} \mathrm{C}$ for $16 \mathrm{~h}$. The dried mycelium biomass ( 3 parallel samples with the same condition) was weighed and transformed for comparative analyses.

Table 1. Water Contents and Added Amounts of Wood Vinegar Samples

\begin{tabular}{|c|c|c|c|c|c|c|}
\hline & A1 & A2 & A3 & A4 & A5 & A6 \\
\hline Water Content $(\%)$ & 91.52 & 93.37 & 94.97 & 93.89 & 83.11 & 91.19 \\
\hline Added Amount $(\mu \mathrm{L})$ & 10.00 & 12.79 & 16.86 & 13.88 & 5.02 & 9.63 \\
\hline & P1 & P2 & P3 & P4 & P5 & P6 \\
\hline Water Content $(\%)$ & 92.19 & 92.91 & 94.63 & 94.12 & 82.71 & 90.88 \\
\hline Added Amount $(\mu \mathrm{L})$ & 10.00 & 11.02 & 14.54 & 13.28 & 4.52 & 8.56 \\
\hline
\end{tabular}




\section{Design of Experiment}

The Historical Date Design (HDD) (Longley et al. 1967) was used to assess the influences, both individually and interactively, of the wood vinegar components on the mycelium growth. The components of each sample were analyzed by GC-MS and then categorized into 7 groups accordingly, as shown in Table 2.

Table 2. Classification of Chemical Groups in Wood Vinegar Samples

\begin{tabular}{|c|c|c|c|}
\hline \multirow[b]{2}{*}{$\begin{array}{c}\text { Chemical } \\
\text { Groups }\end{array}$} & \multirow[b]{2}{*}{ Compounds } & \multicolumn{2}{|c|}{ Component Content of Compounds (\%) } \\
\hline & & $\begin{array}{c}\text { Crude Apple Wood } \\
\text { Vinegar }\end{array}$ & $\begin{array}{c}\text { Crude Pear Wood } \\
\text { Vinegar }\end{array}$ \\
\hline \multirow[t]{6}{*}{ Acids } & Acetic Acid & 46.24 & 48.11 \\
\hline & Propionic Acid & 0.92 & 1.20 \\
\hline & 2-Methyl Propionic Acid & 0.42 & 0.20 \\
\hline & Butyric Acid & 0.20 & / \\
\hline & Hexanoic Acid & 0.41 & 0.09 \\
\hline & $\begin{array}{l}\text { 3-Methoxy-4-Hydroxyphenyl } \\
\text { Acetic Acid }\end{array}$ & 4.12 & 3.51 \\
\hline \multirow[t]{10}{*}{ Phenols } & Phenol & 0.03 & l \\
\hline & 2-Methyl Phenol & 3.80 & 3.50 \\
\hline & 3-Methyl Phenol & 0.91 & 0.49 \\
\hline & 4-Methyl-2-Methoxy Phenol & 0.76 & 0.84 \\
\hline & 1,2-Benzenediol & 4.62 & 4.55 \\
\hline & 4-Ethyl-2-Methoxy Phenol & 2.22 & 2.58 \\
\hline & 2-Methoxy-4-(2-Propenyl)Phenol & 0.43 & 0.41 \\
\hline & 4-Propenyl-2-Methoxy Phenol & 0.27 & 0.45 \\
\hline & 4-Propyl-2-Methoxy Phenol & 0.30 & 0.32 \\
\hline & 4-Hydroxyalkyl-2-Methoxy Phenol & 0.12 & 0.10 \\
\hline \multirow[t]{9}{*}{ Ketones } & Hydroxyacetone & 10.21 & 8.86 \\
\hline & 2-Furanone & 1.93 & 1.45 \\
\hline & Acetoin & 1.43 & 0.92 \\
\hline & 2,4-Pentanedione & 0.71 & 0.42 \\
\hline & 3-Methyl-2-Cyclopenten-1-One & 0.78 & 0.36 \\
\hline & Methyl Cyclopentenolone & 2.95 & 2.37 \\
\hline & 4-Methylcyclohexanone & 0.62 & 1.05 \\
\hline & acetone & 0.90 & 0.13 \\
\hline & 7-Tridecanone & 1.29 & 1.36 \\
\hline \multirow[t]{5}{*}{ Aldehydes } & Furfural & 0.58 & 5.52 \\
\hline & 5-Methyl-2-Furfural & 0.20 & 0.31 \\
\hline & 2-Ethyl Hexanal & 0.82 & 0.94 \\
\hline & 5-Hydroxymethyl Furfural & l & 0.39 \\
\hline & Vanilline & 0.30 & 0.81 \\
\hline \multirow[t]{2}{*}{ Esters } & Formic Acid, Tetrahydrofuryl Eater & 0.91 & 0.05 \\
\hline & $\begin{array}{l}\text { 2-Methyl-1-Propenoic Acid, Ethyl } \\
\text { Eater }\end{array}$ & 1.61 & 0.79 \\
\hline \multirow[t]{4}{*}{ Alcohols } & 2-Furfuryl Alcohol & 0.33 & 0.31 \\
\hline & $\begin{array}{l}\text { 4-Hydroxy-3-Methoxyphenethyl } \\
\text { Alcohol }\end{array}$ & 0.40 & / \\
\hline & Furfuryl Alcohol & 2.69 & 2.98 \\
\hline & Isosorbide & 2.57 & 1.03 \\
\hline Others & Unidentified & 2.99 & 3.60 \\
\hline
\end{tabular}

The seven groups were organic acids, phenols, alcohols, ketones, aldehydes, esters, and others unidentified. In HDD, the samples were divided into two blocks as a mathematical approach to removing unidentifiable variation caused by differences between the species of the raw materials. Specifically, block 1 was for apple wood vinegar 
components, and block 2 was for pear wood vinegar components (Table 3). The data analysis was performed using Design Expert Software 11.0.4.0 (Stat-Ease, Inc., Minneapolis, MN, USA).

Table 3. HDD of Components' Effects on Mycelium Growth

\begin{tabular}{|c|c|c|c|c|c|c|c|c|}
\hline $\begin{array}{c}\text { Block } \\
\text {-run }\end{array}$ & $\begin{array}{c}\text { Acids } \\
(\mathrm{A})(\%)\end{array}$ & $\begin{array}{c}\text { Phenols } \\
(\mathrm{B})(\%)\end{array}$ & $\begin{array}{c}\text { Ketones } \\
(\mathrm{C})(\%)\end{array}$ & $\begin{array}{c}\text { Aldehydes } \\
(\mathrm{D})(\%)\end{array}$ & $\begin{array}{c}\text { Esters } \\
(\mathrm{E})(\%)\end{array}$ & $\begin{array}{c}\text { Alcohols } \\
(\mathrm{F})(\%)\end{array}$ & $\begin{array}{c}\text { Others } \\
(\mathrm{G}) \\
(\%)\end{array}$ & $\begin{array}{c}\text { Res. } \\
(\%)\end{array}$ \\
\hline 1-A1 & 52.31 & 13.46 & 20.82 & 1.90 & 2.52 & 5.99 & 2.99 & 89 \\
\hline 1-A2 & 53.03 & 14.63 & 17.67 & 10.09 & 2.36 & 2.01 & 0.21 & 95 \\
\hline 1-A3 & 46.96 & 4.83 & 28.66 & 13.76 & 3.52 & 0.06 & 2.21 & 103 \\
\hline 1-A4 & 84.25 & 1.80 & 13.65 & 0.01 & 0.00 & 0.00 & 0.30 & 133 \\
\hline 1-A5 & 59.37 & 13.27 & 1.21 & 0.03 & 9.98 & 0.00 & 16.14 & 185 \\
\hline 1-A6 & 59.62 & 16.99 & 12.78 & 6.44 & 0.00 & 4.17 & 0.00 & 222 \\
\hline 2-P1 & 53.11 & 13.24 & 16.92 & 7.97 & 0.84 & 4.32 & 3.60 & 95 \\
\hline 2-P2 & 53.72 & 15.69 & 17.80 & 6.95 & 3.46 & 2.32 & 0.06 & 99 \\
\hline 2-P3 & 47.30 & 6.32 & 20.13 & 17.84 & 0.87 & 0.12 & 7.42 & 105 \\
\hline 2-P4 & 83.99 & 2.59 & 10.92 & 0.09 & 0.00 & 0.80 & 1.61 & 135 \\
\hline 2-P5 & 55.01 & 7.43 & 7.36 & 0.38 & 13.02 & 4.35 & 12.45 & 190 \\
\hline 2-P6 & 62.65 & 16.11 & 12.20 & 5.58 & 0.27 & 0.00 & 3.19 & 239 \\
\hline
\end{tabular}

${ }^{*}$ Res. - Response variable

\section{RESULTS AND DISCUSSION}

\section{Overall Effects of Wood Vinegar on Mycelium Growth}

Wood vinegars show growth-enhancing effects on various kinds of edible fungi (Zhang 1991; Yoshimura and Hayakawa 1991). According to Ni et al. (2011), adding $0.05 \%$ of wood vinegar supernatant into oyster mushroom and black forest mushroom cultivation compounds led to significant mycelium growth rate increases of $13.50 \%$ and $12.67 \%$, for each strain respectively. Additionally, the same study confirmed that both the total content of amino acids and the content of essential amino acids showed no significant influence over the growth-enhancing effects. Enhancing effects were also observed in the preliminary stage of the present study at both concentrations of $0.05 \%$ and $0.1 \%$. Specifically, using the refining and preparing process described earlier in this article, the two wood vinegar samples in the test were divided into 6 groups in each block, as shown in Table 3. A total of 12 pretreated wood vinegar samples with distinctive chemical compositions were added to the culturing medium to assess the influence of each sample on mycelium growth. The results are depicted in Fig. 1 and were statistically tested for significance as shown in Table 4. Remarkably, with a confidence level of 95\%, all the samples in the test showed significant differences from each other. In addition, all samples except A2 and P2 showed significant differences from the control group. Specifically, the growth rates of A1 and P1 were less than the control, while the rest of the samples (A3 to $\mathrm{A} 6$ and $\mathrm{P} 3$ to P6) were greater than the control, ranging from $103 \%$ to $239 \%$. More rigorous inspection with a confidence level of $99 \%$ indicated that A1, A2, P1, P2, and P3 showed no significant differences from the control. Notably, A6 and P6 were the most effective components in terms of mycelium growth enhancement.

In general, the results suggested that, at the concentration of $0.1 \mathrm{vol} \%$, the influence of wood vinegar supernatants (treated with active carbon or not) on mycelium growth was not rigorously evident $(p<0.01)$. Conversely, instead of enhancing the mycelium growth, 
as suggested in some studies, the supernatants A1, A2, P1, and P2 showed the potential to slightly inhibit the growth $(\mathrm{p}<0.05)$. However, by a 3 -stage reduced pressure distillation at different temperature intervals, all three distillated fractions showed significant $(\mathrm{p}<$ 0.01 ) enhancing effects on mycelium growth rates, and greater distillation temperatures resulted in greater enhancing effects. Comparing A3 and P3 (distilled under $97^{\circ} \mathrm{C}$ ) with A5 and P5 (distilled at $100{ }^{\circ} \mathrm{C}$ to $105^{\circ} \mathrm{C}$ ), the mycelium-enhancing effects increased by approximately $80 \%$ as the distillation temperature increased. Thus, at this stage it was reasonable to infer from the experiment the following: Firstly, substance(s) with growthenhancing effects were present in the wood vinegar supernatants. Secondly, there was/were also negative substance $(\mathrm{s})$ that could neutralize $(\mathrm{p}<0.01)$ the growth-enhancing effects or even inhibit $(\mathrm{p}<0.05)$ the mycelium growth. Thirdly, compared with the enhancing substance(s), the inhibiting substance(s) had (a) lower boiling point(s) and could be effectively separated from the enhancing component(s) by ethyl acetate extraction of the latter. The findings could provide an explanation for the contradictions among previous studies. However, identification of the active chemicals, either enhancing or inhibiting, requires a considerable amount of data analyzed at the molecular level in response to the growing conditions of the mushrooms. Limited by time and experimental conditions, the information required is not given in adequate detail in this study for further statistical inference. Future works in this direction not only should focus on the separation and refinement of certain wood vinegar samples but also pay attention to the carbonization process when the wood vinegar precursors and components are accumulated in the furnace flue. Thus, abundant variations of chemical concentration gradients could be achieved and then assigned as independent variables by further experiment designs. By collection of adequate data and by employing the method described in this study, it will be feasible to settle the present uncertainties regarding the mechanisms by which wood vinegar affects fungal growth. It is worth mentioning, however, that the mushroom growing conditions in future studies should also be recorded in blocks of individual stains of fungi. This is because, to the knowledge of the authors, the chemical group sensitivity differs from strain to strain even within the same species, but the variation tendency is generally about the same.

Table 4. Effects of Pretreated Wood Vinegar on Mycelium Growth

\begin{tabular}{|c|c|c|c|c|c|}
\hline \multirow[t]{2}{*}{ Sample } & \multirow{2}{*}{$\begin{array}{l}\text { Mycelium } \\
\text { Growth } \\
\text { (mg/flask) }\end{array}$} & \multirow{2}{*}{$\begin{array}{c}\text { Growth Rate } \\
(\%)\end{array}$} & \multirow{2}{*}{$\begin{array}{l}\text { Standard } \\
\text { Deviation } \\
\text { (mg/flask) }\end{array}$} & \multicolumn{2}{|c|}{$\begin{array}{c}\text { Significance } \\
\text { (t-test) }\end{array}$} \\
\hline & & & & $p<0.05$ & $p<0.01$ \\
\hline Control & 25.20 & 100 & 0.51 & ee' & ED'E' \\
\hline $\mathrm{A} 1$ & 22.43 & 89 & 0.84 & $f$ & $E$ \\
\hline A2 & 23.94 & 95 & 0.63 & $\mathrm{e}$ & $E$ \\
\hline A3 & 25.96 & 103 & 0.38 & $d$ & $\bar{D}$ \\
\hline A4 & 33.52 & 133 & 0.24 & C & C \\
\hline A5 & 46.62 & 185 & 0.96 & $\mathrm{~b}$ & $B$ \\
\hline A6 & 55.94 & 222 & 0.45 & $a$ & A \\
\hline $\mathrm{P} 1$ & 23.94 & 95 & 0.39 & $f^{\prime}$ & $E^{\prime}$ \\
\hline P2 & 24.95 & 99 & 0.58 & $\mathrm{e}^{\prime}$ & $E^{\prime}$ \\
\hline P3 & 26.46 & 105 & 0.65 & $d^{\prime}$ & $\bar{D}^{\prime}$ \\
\hline P4 & 34.02 & 135 & 0.55 & $c^{\prime}$ & $\mathrm{C}^{\prime}$ \\
\hline P5 & 47.88 & 190 & 0.39 & $\mathrm{~b}^{\prime}$ & $\mathrm{B}^{\prime}$ \\
\hline P6 & 60.23 & 239 & 0.37 & $a^{\prime}$ & $A^{\prime}$ \\
\hline
\end{tabular}




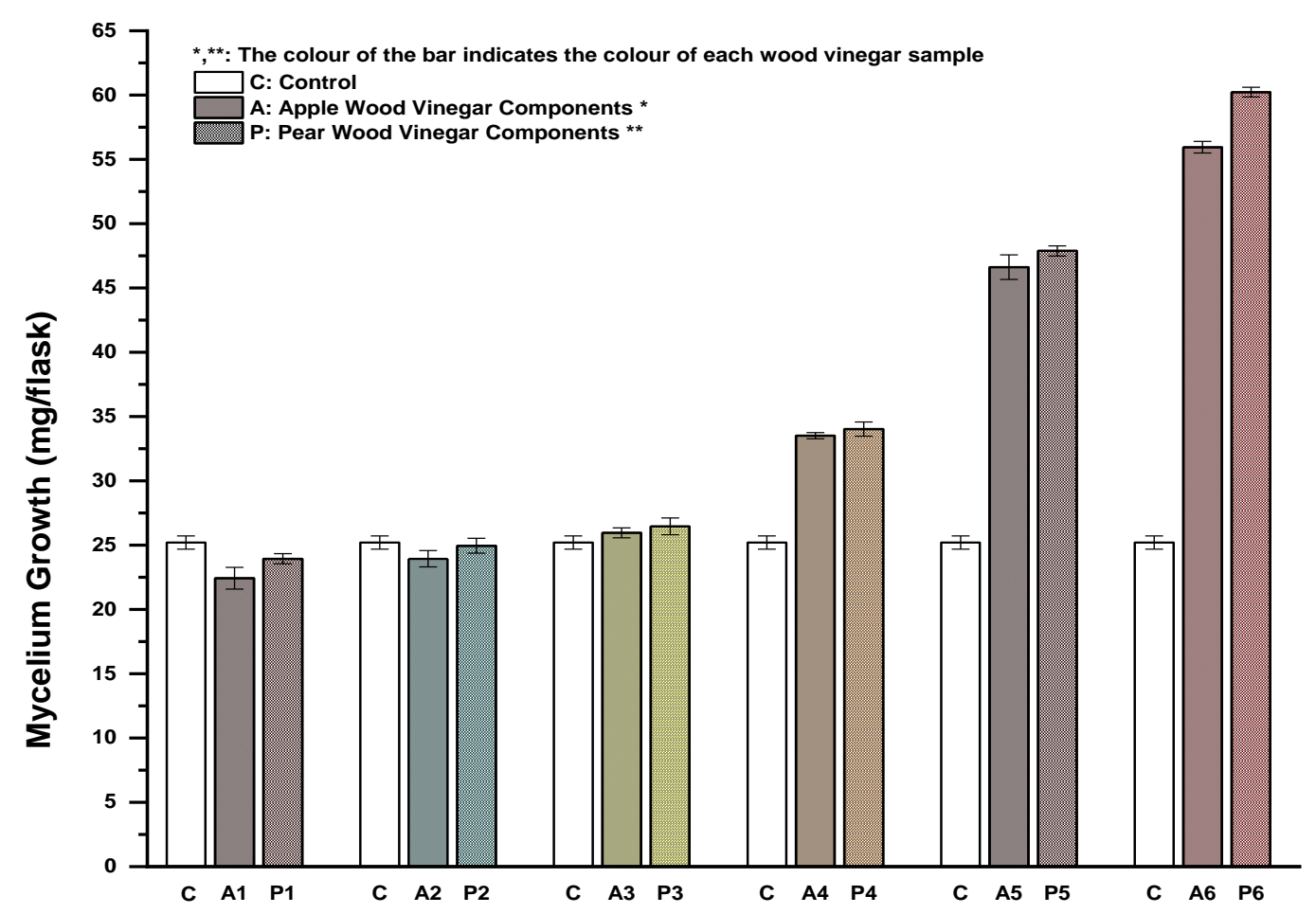

Fig. 1. Effects of pretreated wood vinegar on mycelium growth

\section{Synergistic Effects among Wood Vinegar Components}

While the active effects of wood vinegar components on mycelium growth were confirmed in this study, it is not prudent at this stage to deduce the individual identities of active components with the information acquired heretofore. However, it is feasible to make statistical inferences of the roles played by the groups of chemicals. By the classification shown in Table 3, almost all chemicals (>99.5\%) in the wood vinegar samples could be separated into 7 groups (acids (A), phenols (B), ketones (C), aldehydes (D), esters (E), alcohols (F), and others $(\mathrm{G})$ ) to formulate Scheffé mix models (Piepel et al. 2002), which were specifically designed to handle natural constrains in complementary mixtures. The seven groups of chemicals were formulated into a quadratic model (Eq. 1), where $Y$ is a response representing relative mycelium growth rates in comparison with the control, and $\beta_{\mathrm{i}}$ and $\beta_{\mathrm{ij}}$ are, respectively, linear and quadratic coefficients.

$$
Y=\sum_{i=1}^{p} \beta_{i} x_{i}+\sum \sum_{i<j}^{p} \beta_{i j} x_{i} x_{j}
$$

Equation 1 was used as selection algorithm with a confidence level of $90 \%$. As a result, the seven basic chemicals groups and two interactive terms were found significant and selected by the algorithm. The equation coefficients and confidence intervals of the selected terms are shown in Table 5. To make a more explicit prediction of mycelium growth for a given level of a chemical group, the equation coefficients were transformed into actual units as a percentage of mycelium growth rate, and the actual equation is shown as Table 6. According to Table 6, the phenols group was the most likely to be the main cause of inhibiting effects; notably, this inference aligns with the conclusion drawn in the previous section. According to the GC-MS analyses, the major components of the phenols group in the wood vinegars were 1,2-benzenediol (17.7\%), 2-methyl phenol (15.2\%), and 4-ethyl-2-methoxyphenol (13.9\%), which, as predicted, have relatively high boiling points 
and greater solubility in water than in ethyl acetate. The real equation in Table 7 also showed lesser inhibiting groups, such as ketones and acids. Chemicals in those groups are well known as metabolic inhibitors, such as acetone (accounting for $22.2 \%$ of the ketones group) and acetic and hexanoic acids (accounting for $28.2 \%$ and $48.9 \%$ of the acids group, respectively). Interestingly, the interactive terms of acids with phenols and acids with acetones were positive in the real equation, indicating the existence of synergistic growthenhancing effects among the two pairs (AB and $\mathrm{AC}$ ) of those chemical groups. The ANOVA tests (Table 5) confirmed the above effects as significant. The linear mixture term represents the effects of each chemical group reacted individually, while the $A B$ and $A C$ terms represent the interactive effects of acids with phenols and acids with acetones. Overall, the significances of the model and its terms were satisfactory. Notably, however, the p-value of term AC was slightly greater than 0.05 . There are two reasons behind this: First, the mycelium growth rate, which was used in this study as the response variance, was not as sensitive to ketones as to other chemical groups. Second, the concentration gradients of the ketones group were not differentiated ideally in the tests, resulting in multilinearity. Still, with an F-value of 13.62 and corresponding p-value of 0.065 , it was reasonable that the synergistic enhancing effect was induced by intergroup difference by term AC rather than intragroup errors. Cautiously, Student's t-test was applied on the coefficients of the terms to verify their effects on the response at confidence levels of $95 \%$ and $90 \%$ (Table 5 ). The results showed that the signs of three terms (namely, A, G, and AC) were uncertain at the $95 \%$ confidence level but were determined at the $90 \%$ confidence level. Therefore, the results validated the hypothesis that there were synergistic effects among acids and phenols and among acids and ketones, and by these effects the individually inhibiting chemicals interacted synergistically as mycelium growth promoters.

Table 5. ANOVA for Reduced Quadratic Model

\begin{tabular}{|c|c|c|c|c|c|}
\hline Source & Sum of Squares & df & Mean Square & F-value & p-value \\
\hline Model & 31830.25 & 8 & 3978.78 & 76.95 & 0.0129 \\
\hline Linear Mixture & 15863.54 & 6 & 2643.92 & 51.13 & 0.0193 \\
\hline AB & 15813.43 & 1 & 15813.43 & 305.83 & 0.0033 \\
\hline AC & 704.02 & 1 & 704.02 & 13.62 & 0.0662 \\
\hline Residual & 103.41 & 2 & 51.71 & & \\
\hline Block & 108.00 & 1 & 108.00 & & \\
\hline Cor Total & 32041.67 & 11 & & & \\
\hline
\end{tabular}

Table 6. Coefficients of Equation in Terms of Components

\begin{tabular}{|c|c|c|c|c|c|c|}
\hline Term & $\begin{array}{c}\text { Coefficient } \\
\text { Estimate }\end{array}$ & $\begin{array}{c}\text { Standard } \\
\text { Error }\end{array}$ & $\begin{array}{c}95 \% \mathrm{Cl} \\
(\mathrm{Low})\end{array}$ & $\begin{array}{c}95 \% \mathrm{Cl} \\
(\mathrm{High})\end{array}$ & $\begin{array}{c}90 \% \mathrm{Cl} \\
(\mathrm{Low})\end{array}$ & $\begin{array}{c}90 \% \mathrm{Cl} \\
\text { (High) }\end{array}$ \\
\hline $\mathrm{A}$ & -180.36 & 61.55 & -445.17 & 84.45 & -360.07 & -177.44 \\
\hline $\mathrm{B}$ & -7844.93 & 472.83 & 9879.35 & 5810.51 & 9225.59 & 7842.01 \\
\hline $\mathrm{C}$ & -1322.78 & 388.80 & 2995.65 & 350.10 & 2458.08 & 1319.86 \\
\hline $\mathrm{D}$ & 878.52 & 105.96 & 422.63 & 1334.42 & 569.13 & 881.44 \\
\hline $\mathrm{E}$ & 1015.21 & 126.94 & 469.03 & 1561.40 & 644.54 & 1018.13 \\
\hline $\mathrm{F}$ & 1169.78 & 160.70 & 478.33 & 1861.22 & 700.53 & 1172.70 \\
\hline $\mathrm{G}$ & 490.09 & 137.52 & -101.62 & 1081.80 & 88.53 & 493.01 \\
\hline $\mathrm{AB}$ & 14477.92 & 827.88 & 10915.85 & 18039.99 & 12060.52 & 14480.84 \\
\hline $\mathrm{AC}$ & 3322.45 & 900.41 & -551.68 & 7196.58 & 693.26 & 3325.37 \\
\hline
\end{tabular}

$\mathrm{Cl}$ - confidence interval 
Table 7. Actual Equation for Reduced Quadratic Model

\begin{tabular}{|c|c|}
\hline$Y$ (Mycelium Growth Rate ) $(\%)$ & $=$ \\
\hline-1.80359 & Acids $(A)(\%)$ \\
\hline-78.44926 & Phenols $(B)(\%)$ \\
\hline-13.22777 & Ketones $(C)(\%)$ \\
\hline+8.78522 & Aldehydes $(D)(\%)$ \\
\hline+10.15214 & Esters $(E)(\%)$ \\
\hline+11.69777 & Alcohols $(F)(\%)$ \\
\hline+4.90093 & Others $(G)(\%)$ \\
\hline+1.44779 & Acids $(A){ }^{*}$ Phenols $(B)(\%)$ \\
\hline+0.332245 & Acids $(A){ }^{*}$ Ketones $(C)(\%)$ \\
\hline
\end{tabular}

\section{CONCLUSIONS}

1. By a combination of separating and refining methods, two kinds of original wood vinegars were separated into two groups with six samples in each. Adding the separated samples into the mycelium-culturing media revealed the presence and characteristics of enhancing and inhibiting chemicals in the original wood vinegars. The enhancing groups of chemicals were (ranked by effects) alcohols $>$ esters $>$ aldehydes; the inhibiting groups of chemicals were (ranked by effects) phenols $>$ ketones $>$ acids.

2. Chemical and statistical inferences indicated that the principle inhibitory chemicals in the wood vinegars were most likely 1,2-benzenediol, 2-methyl phenol, and 4-ethyl-2methoxyphenol.

3. Analysis of variance revealed synergistic effects between acids and phenols and between acids and ketones. By these effects, the inhibiting chemicals interacted synergistically as mycelium growth promoters.

\section{ACKNOWLEDGMENTS}

This work was funded by the National Key R\&D Program of China (Grant No. 2017YFD0601002). The authors declare that they have no conflicts of interest.

\section{REFERENCES CITED}

Burnette, R. (2010). "An introduction to wood vinegar," (http://c.ymcdn.com/sites/www.echocommunity.org), Accessed 13 November 2019.

Ikegaya, N., Goto, M., and Hayashi, Y. (1994). "Effect of phenolic compounds and urovides on the activities of extracellular enzyme during vegetative growth and fruitbody formation of Lentinus edodes," Transactions of the Mycological Society of Japan 34, 195-207.

Longley, J. W. (1967). "An appraisal of least squares programs for the electronic computer from the point of view of the user," Journal of the American Statistical Association 62(319), 819-841.

Ni, S., Zhang, H., Sun, L., Wang, Y., and Ping, A. (2011). "Effect of pyroligneous acid on oyster mushroom and black forest mushroom production," Heilongjiang Agricultural 
Sciences 7(1), 42-49.

Piepel, G. F., Szychowski, J. M., and Loeppky, J. L. (2002). "Augmenting Scheffé linear mixture models with squared and/or crossproduct terms," Journal of Quality Technology 34(3), 297-314. DOI: 10.1080/00224065.2002.11980160

Singh, V. P., Singh, G., Kumar, B., Kumar, A., and Srivastava, S. (2018). "Effect of various chemicals on the mycelial growth and fruiting body of milky mushroom (Calocybe indica)," Asian Journal of Crop Science 10(4), 168-173. DOI: 10.3923/ajcs.2018.168.173

Ghose, T. K. (1987). "Measurement of cellulase activities," Pure and Applied Chemistry 59(2), 257-268.

Wu, Q., Zhang, S., and Hou, B. (2015). "Study on the preparation of wood vinegar from biomass residues by carbonization process," Bioresource Technology 179, 98-103. DOI: 10.1016/j.biortech.2014.12.026

Yatagai, M., Nishimoto, M., Hori, K., Ohira, T., and Shibata, A. (2002). "Termiticidal activity of wood vinegar, its components and their homologues," Journal of Wood Science 48(4), 338-342. DOI: 10.1007/BF00831357

Yoshimura, H., and Hayakawa, T. (1991). "Acceleration effect of wood vinegar from Quercus crispula on the mycelial growth of some basidiomycetes," Transactions of the Mycological Society of Japan 32, 55-64.

Yoshimura, H., Washio, H., Yoshida, S., Seino, T., Otaka, M., Matsubara, K., and Matsubara, M. (1995). "Promoting effect of wood vinegar compounds on fruit-body formation of Pleurotus ostreatus," Mycoscience 36(2), 173-177. DOI: 10.1007/BF02268554

Zhang, L. J. (1991). "Studies on wood tar (Wt-I) as an additive for increasing output of edible fungi," Edible Fungi of China 4(1), 1-5.

Article submitted: November 25, 2019; Peer review completed: February 1, 2020;

Revised version received: February 25, 2020; Accepted: February 28, 2020; Published: March 13, 2020.

DOI: 10.15376/biores.15.2.2961-2970 